\title{
Aplicação da Análise de Estabilidade de von Neumman a Problemas Transientes de Natureza Parabólica
}

Pinheiro, V. P. ${ }^{1}$; Loeffler, C.F. ${ }^{1}$; Neves, N. S. ${ }^{2}$; Bodart, N.L.O. ${ }^{3}$; Almeida, L.M $^{3 *}$; Agrizzi, V. ${ }^{3}$; Pinheiro, L.P. ${ }^{4}$;

1 Programa de Pós-Graduação em Engenharia Mecânica, Universidade Federal do Espírito Santo, Vitória, ES, Brasil.

2 Programa de Pós-Graduação em Engenharia Civil, Universidade Federal do Espírito Santo, Vitória, ES, Brasil.

3 Programa de Iniciação Científica em Métodos Numéricos, Universidade Vila Velha, Vila Velha, ES , Brasil.

4 Departamento de Engenharia Mecânica, Instituto Federal do Espírito Santo, Vitória, ES, Brasil.

* e-mail: larissa.m.de.almeida@gmail.com

\section{Resumo}

A solução numérica de equações diferenciais parciais transientes é frequentemente demandada em análises de modelos matemáticos de campo escalar presentes na Engenharia. Neste contexto, pretende-se no vigente artigo, restrito a equações de natureza parabólica, destacar a importância do conceito de critério de estabilidade para discretizações temporais que utilizam o método de Euler explícito. Um entendimento mais consolidado da Análise de Estabilidade de von Neumann auxilia na dedução de critérios de estabilidade para diversos problemas lineares mais simples, e adicionalmente, como paralelo para análises de estabilidade não-analíticas de fenômenos descritos por modelos não-lineares.

Palavras chave: Equações Diferenciais Parciais, Critério de Estabilidade, Análise de Estabilidade de Fourier

\begin{abstract}
The numerical solution of transient partial differential equations is often required in order to analyze mathematical models of scalar field present in Engineering. Hence the current article aims to highlight the importance of the concept of stability criterion in temporal discretizations using the Euler explicit method, while focusing solely on parabolic equations. A comprehensive understanding of the von Neumann Stability Analysis contributes in the deduction of stability criteria for several simpler linear problems, and additionally, as a parallel for non-analytical stability analyses of phenomena described by nonlinear models.
\end{abstract}

Keywords: Partial Differential Equations, Stability Criterion, Fourier Stability Analysis.

\section{Introdução}

No contexto de modelos matemáticos descritivos de fenômenos físicos relevantes à engenharia, são de essencial importância aqueles com natureza de marcha no tempo [1], e por sua vez, passíveis de aplicações dos mais diversos métodos numéricos. Dentre tais técnicas, destaca-se a das diferenças finitas por sua eficiência e simplicidade na solução de problemas lineares [2]. Há portanto diversas formas de abordar a discretização do domínio do tempo, dentre as quais o método explícito de Euler é aqui selecionado, justamente por sua característica 


\section{Blucher Proceedings X Encontro Científico de Física Aplicada

condicionalmente estável [3].

$\mathrm{O}$ vigente artigo tem como viés central a exposição sistêmica da análise de estabilidade de von Neumann, utilizada para deduzir de forma matemática fechada, expressões de critério de estabilidade para problemas lineares. Para tanto, seleciona-se uma equação diferencial parcial de natureza parabólica e unidimensional no domínio espacial para uma exposição didática e direta do procedimento.

\section{Equações de Governo}

Em uma gama vasta de modelos matemáticos utilizados em Engenharia, destacam-se aqueles derivados da Teoria de Campo Escalar Generalizada. Problemas físicos onde visa-se o conhecimento do comportamento de campos escalares são frequentes e muitos difundidos na área tecnológica nas mais diversas searas tais como difusão de calor e massa, escoamentos potenciais, percolação dentre muitas outras. Uma forma de representar tal teoria pode ser algebricamente ilustrada pela equação 1 a seguir.

$$
\left[K_{i j} u,_{j}\right]_{, i}-\lambda D^{n}(u)=p
$$

Onde $K_{i j}$ é um diádico de segunda ordem que armazena características físicas do sistema em cada direção, $u$ é o campo potencial escalar de interesse, $\lambda$ é uma constante escalar, $D^{n}(u)$ é uma derivada temporal de ordem $n$ e por fim $p$ caracteriza uma ação de domínio [4].

A natureza geral da equação 1 se dá pela capacidade de representar problemas lineares, não lineares, homogêneos ou não-homogêneos, puramente difusivos ou mesmo com efeitos advectivos [5]. Além disso, com enfoque no termo temporal, a ordem da derivada temporal define a natureza parabólica ou hiperbólica em relação ao domínio do tempo [6].

Agora em um contexto mais simples, considera-se um problema puramente difusivo de natureza parabólica no tempo e unidimensional no espaço. Tal fenômenos pode ser representado satisfatoriamente com o modelo matemático que segue.

$$
\frac{\partial u}{\partial t}=\alpha \frac{\partial^{2} u}{\partial x^{2}}
$$

$\mathrm{Na}$ equação 2 , o campo potencial é dado por $u$ e $\alpha$ representa uma constante física do fenômeno. Pode-se então utilizar aproximações de diferenças finitas centrais para do domínio espacial e tratar a derivada temporal de forma adiantada, o que caracteriza, por sua vez, o método de Euler explícito [2], para gerar a equação discreta de diferença finita que segue.

$$
\frac{u_{i}^{n+1}-u_{i}^{n}}{\Delta t}=\alpha \frac{u_{i-1}^{n}-2 u_{i}^{n}+u_{i+1}^{n}}{(\Delta x)^{2}}
$$

\section{Análise de Estabilidade de von Neumann}

A estabilidade de um método numérico, no contexto de problemas transientes, pode ser caracterizada por uma limitação razoável dos erros ou perturbações presentes em cada passo no tempo, de forma a manter o comportamento da solução controlado, evitando que os módulos e as variáveis cresçam ilimitadamente [3].

A natureza de estabilidade de cada tipo de discretização temporal é distinta, onde destaca-se aqui o método de Euler explícito, sob análise, que consiste em procedimento simples, entretanto, condicionalmente estável para lidar com problemas de marcha no tempo. No caso deste tipo de discretização temporal, a determinação de um critério de estabilidade se faz necessário, justamente para determinar uma faixa de atuação consistente do método de diferenças finitas.

A abordagem mais usual sobre critérios de estabilidade consiste em sua dedução via analogia física do problema em questão. Tais abordagens são suficientemente precisas para alguns proble- 


\section{Blucher Proceedings X Encontro Científico de Física Aplicada

mas, mas insuficientes ou não assertivas para outros modelos, gerando a demanda pelo uso de uma técnica com maior fundamentação matemática. Neste tocante, objetiva-se aqui a exposição do procedimento de análise de Estabilidade de von Neumann, para determinação de critérios de estabilidade em problemas físicos regidos por equações diferenciais lineares.

O cerne da análise consiste na reescrita de cada valor dos campos potenciais da equação de diferenças finitas 3 , em formato de série de Fourier composta por exponenciais complexas [8]. Tal aproximação podo ser algebrizada na equação 4 a seguir.

$$
u_{j}^{n}=\sum_{j} e^{\gamma n \Delta t} e^{i \beta_{m} j \Delta x}
$$

A nível de detalhamento, uma série de Fourier tradicional é constituída por funções harmônicas ortogonais entre si, tal como na equação 5 sequencialmente, onde as constantes $a_{0}, a_{n}, b_{n}$ podem ser determinadas sem maiores dificuldades utilizando condições de ortogonalidade [1], tal como o número de onda $\beta_{m}$ dependente do período $L$ da série.

$$
\begin{gathered}
f(x)=a_{0}+\sum_{n=1}^{\infty}\left(a_{n} \cos \beta_{m} x+b_{n} \sin \beta_{m} x\right) \\
\beta_{m}=\frac{n \pi}{L}
\end{gathered}
$$

É possível utilizar a Fórmula de Euler, equação 6, com algumas manipulações, para reescrever as funções harmônicas da séries de Fourier clássica da equação 5, gerando a possibilidade de reescrever a série em termos de exponenciais de argumento complexo, tal como mostrado a priori pela equação 4 , que pode ser utilizada como base para análises de estabilidade de modelos diferenciais lineares e não-lineares.

$$
e^{\beta_{m} x i}=\cos \left(\beta_{m} x\right)+i \sin \left(\beta_{m} x\right)
$$

Se tratando de modelos diferenciais lineares com coeficientes constantes, numa abordagem mais simplista, é possível executar toda a análise fundamentada apenas em um único termo da série de Fourier, fato este embasado pela validade do princípio da superposição de efeitos neste caso específico. Com isso, extraindo este termo de base e definindo uma nova variável $\xi$ abaixo na expressão 7, denominada como amplitude e valendo-se de sua definição matemática, pode-se reescrever o termo base tal como na equação 8 .

$$
\begin{gathered}
\xi=e^{\gamma \Delta t} \\
u_{j}^{n}=\xi^{n} e^{i \beta_{m} j \Delta x}
\end{gathered}
$$

Pode-se utilizar a equação 8 para reescrever o valor dos campos potenciais em posições espaciais e passos no tempo distintos, e substitui-los na equação de diferenças finitas 3, e com algum algebrismo isolar a variável de amplitude $\xi$, gerando a equação 9 , em que $r$ representa um condensado de variáveis ligado as propriedades físicas do problema tal como à discretização temporal e espacial.

$$
\begin{gathered}
\xi=\frac{u_{j}^{n+1}}{u_{j}^{n}}=1-2 r\left[1-\cos \left(\beta_{m} \Delta x\right)\right] \\
r=\frac{\alpha \Delta t}{(\Delta x)^{2}}
\end{gathered}
$$

É interessante observar na equação 9, que proveniente da manipulação algébrica, temos que a amplitude $\xi$ é dada pelo quociente entre os valores do campo potencial no próximo instante de tempo e no instante atual. Tal observação elucida de forma mais clara a seguinte imposição de matemática para a estabilidade.

$$
|\xi|=\left|\frac{u_{j}^{n+1}}{u_{j}^{n}}\right| \leq 1
$$

Por fim, inserindo tal condição dada pela equação 10 , na expressão 9 , chega-se após a solução de algumas inequações ao seguinte critério de estabilidade final para a equação de governo 2 . 


$$
r=\frac{\alpha \Delta t}{(\Delta x)^{2}} \leq \frac{1}{2}
$$

\section{Estudo de Caso}

A experimentação numérica baseia-se na análise unidimensional de uma barra de comprimento unitário, sendo discretizado em $n$ pontos com um espaçamento constante $h=0.05$. Nas extremidade da barra são prescritos potenciais de valores iguais $\bar{\phi}_{1}=1^{\circ} C$ e $\bar{\phi}_{n}=0^{\circ} C$, conforme ilustra na Figura 1.

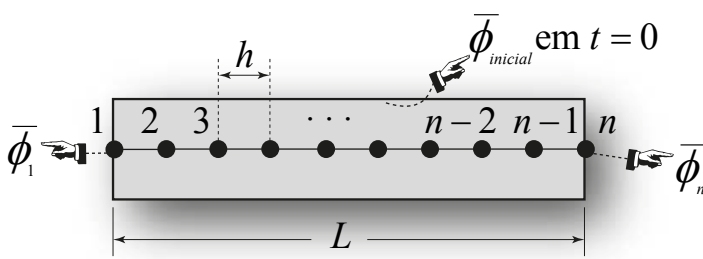

Figura 1: Geometria e condições de contorno

A temperatura da barra no instante inicial $t=0 \mathrm{~s}$ é nula em qualquer posição e a constante $\alpha$ é considerada, por simplicidade, como sendo unitária. Inicialmente, validação é direcionada solução analítica do problema, tal pode ser obtida pelo método de separação de variáveis [1].

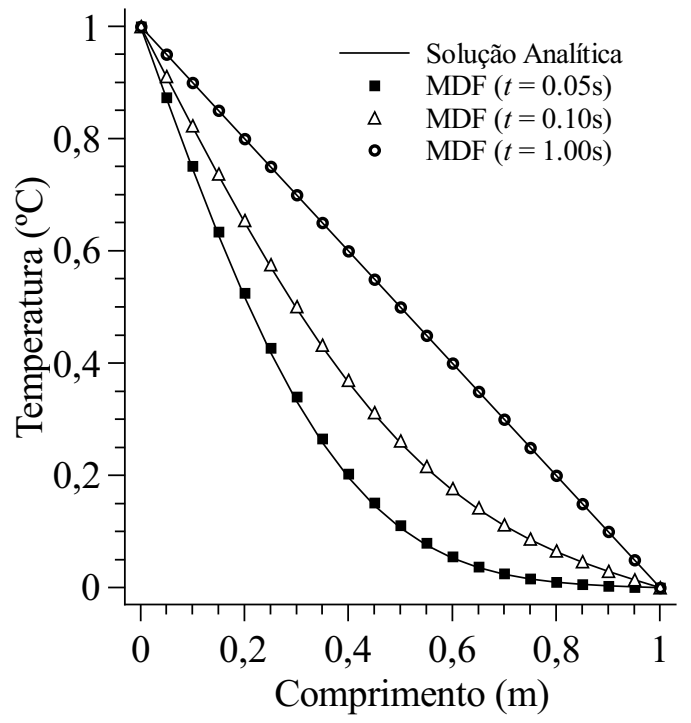

Figura 2: Comparação de resultados com $r=0.4$

Desta forma, a Figura 2 apresenta o comportamento da solução via método das diferenças finitas para distintos instantes de tempos. Com uma rápida análise, é possível verificar que as respostas apresentam-se parelha com a solução de referência. Observa-se ainda, que as curvas respeitam as condições de contorno nas extremidades.

Em adicional, a Figura 3 apresenta uma análise da influência do critério de estabilidade na resposta do problema.

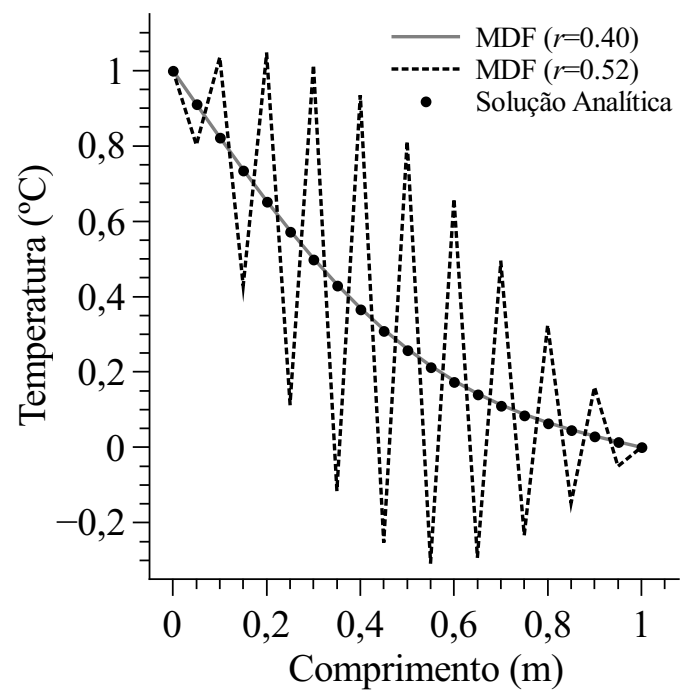

Figura 3: Análise do critério de estabilidade em

$$
t=0.1 s
$$

Nota-se que, a solução que atende o critério de $r \leq 1 / 2$ apresenta um resposta bem ajustada com a solução analítica no tempo de 0.1 segundos. Em contrapartida, uma leve desobediência ao critério, a solução perde estabilidade e apresenta um comportamento oscilatório indesejável.

\section{Considerações Finais}

Com base no estudo de caso, o método explícito de Euler mostra-se uma alternativa numérica conveniente para a solução de equações diferenciais parciais lineares. Destaca-se a importância da validação do critério de estabilidade ao que tange a performance do método explícito, uma vez que, obedecendo o critério de estabilidade, as respostas encontradas se encontram bem ajustadas em comparação à solução analítica. 
Em virtude da simplicidade do método, e seu menor custo computacional, sua aplicação em outros problemas de engenharia é pertinente. A vasta aplicabilidade aliada a seus respectivos critérios de estabilidade, torna-se objeto de pesquisa para futuros projetos.

\section{Referências}

[1] KREYSZIG, E. Matemática Superior para Engenharia. Rio de Janeiro: LTC, 2009.

[2] ANDERSON,D.;TANNEHILL, J.C.;PLETCHER, R.H. Computational Fluid Mechanics and Heat Transfer. CRC Press, 2016.

[3] DE OLIVEIRA FORTUNA, Armando. Técnicas computacionais para dinâminca dos fluidos: conceitos básicos e aplicações. São Paulo: Edusp, 2000.

[4] BULCÃO, A. Formulação do Método dos Elementos de Con- torno com Dupla Reciprocidade usando Elementos de Ordem Superior Aplicada a Problemas de Campo Escalar Generalizado. Dissertação de Mestrado. Programa de Pós-Graduação de Engenharia Mecânica, PPGEM-UFES, Vitória, 1999.

[5] LOEFFLER, C.F. Modelos Mecânicos Derivados da Equação de Campo Escalar Generalizada. Revista Militar de Ciência e Tecnologia, 9, 24-38.

[6] VERSTEEG, H. K; MALALASEKERA, W. An Introduction to Computational Fluid Dynamics: The Finite Volume Method. Pearson Education, 2007.

[7] BUTKOV, E. Física Matemática. Rio de Janeiro: LTC, 2013.

[8] OZISIK, M. N. et al. Finite Difference Methods in Heat Transfer. CRC Press, 2017. 